\title{
Matrix effects and application of matrix effect factor
}

\author{
Wanlong Zhou ${ }^{1}$, Shuang Yang ${ }^{2}$ \& Perry G Wang ${ }^{*}, 1$ \\ ${ }^{1}$ Office of Regulatory Science, CFSAN, US FDA, College Park, MD 20740, USA \\ ${ }^{2}$ Laboratory of Bacterial Polysaccharides, CBER, US FDA, Silver Spring, MD 20993, USA \\ * Author for correspondence: perry.wang@fda.hhs.gov
}

\section{"Matrix effects are major concerns in quantitative LC-MS analysis because they detrimentally affect the accuracy, precision and sensitivity of a method."}

First draft submitted: 20 September 2017; Accepted for publication: 28 September 2017; Published online: 24 November 2017

Although, LC-MS is one of the most sensitive and selective analytical techniques, it often suffers from matrix effects, especially when using ESI for analyzing extracts of complicated matrices [1-3]. Matrix effects are often caused by the alteration of ionization efficiency of target analytes in the presence of co-eluting compounds in the same matrix. Matrix effects can be observed either as a loss in response (ion suppression) or as an increase in response (ion enhancement). Both the ion suppression and enhancement dramatically affect analytical performance of a method [4]. Therefore, matrix effects must be evaluated when validating an LC-MS method. Since, matrix effects were first observed, efforts have been devoted to understanding the mechanisms and minimizing them [5-8]. In this commentary article, the causes of matrix effects and methods for evaluating, minimizing and/or compensating for them are discussed. A novel concept, matrix effect factor (MEF) using stable isotopically labeled internal standards (SIL-ISs) is introduced and its application is presented. Since, SIL-ISs are usually added in the very beginning of the sample preparation procedure to compensate for matrix effects and recovery, the MEF reflects any loss/gain from both the sample preparation and 'conventional' matrix effects caused by co-eluting components during ionization in a mass spectrometer (MS) ion source.

\section{The causes of matrix effect}

Since, matrix effects were first observed for ESI MS in 1990s, several mechanisms have been proposed $[5,6,9,10]$. These include matrix components preventing analyte from gaining access to the charge, competing with analytes to gain charge, interfering with analyte's ability to remain charged in the gas phase, increasing surface tension of droplet or increasing electric resistance. Although, the exact mechanisms of matrix effects are still not fully understood, it has been widely accepted that the co-eluted matrix can alter ionization efficiency of target analytes and influence signal intensity due to the competition for the available charges and for the access to the droplet surface for gas-phase emission during the electrospray process $[7,8]$. Therefore, any process that changes the ionization efficiency and occurs in the liquid phase and gas phase, will cause matrix effects. For example, some studies showed that the presence of interfering compounds at a higher concentration could increase the viscosity and the surface tension of the droplets, which change the efficiency of their formation and evaporation. The changes in liquid phase could result in the alteration of the amount of charged ions in the gas phase. In addition, matrix components or mobile-phase additives that act as ion-pairing reagents usually reduce ionization efficiency and result in low response [9].

Based on the available literature and our experiences, atmospheric pressure chemical ionization (APCI) and atmospheric pressure photoionization (APPI) sources have less matrix effects than an ESI source, because APCI utilizes gas-phase ion-molecule reactions and APPI utilizes hot solvent vapor for desorption. For APCI, the neutral molecular analyte is first transferred into the gas phase through liquid evaporation in a flow of heated gas [11,12], and then ionization occurs by chemical ionization in the gas phase [13]. The main cause of matrix effects in APCI is that the co-eluting matrix compositions influence the efficiency of charge transfer from needle corona discharge 
to the components to be determined. The formation of a solid phase of the analyte itself or the co-precipitation of nonvolatile components present in the matrix can also cause ion suppression [11].

Compared with ESI and APCI, APPI is the most recently introduced form of atmospheric pressure ionization technique for MS application. APPI is mainly used for nonpolar compounds with poor ionization efficiency by ESI or APCI [14]. APPI usually generates more reproducible signals and its ionization is less susceptible to ion suppression due to matrix effects or salt buffers compared with APCI and ESI [15,16]. The main reason for fewer matrix effects in APPI is because it is a more selective atmospheric pressure ionization technique where an analyte is ionized either directly or indirectly through an ionizable dopant using a krypton vacuum ultraviolet lamp source of $10 \mathrm{eV}$ photons [17]. Because the matrix components are often not ionizable by photons at only $10 \mathrm{eV}$, they produce negligible matrix effect under such conditions. However, the matrix components in the gas phase could act as a dopant, increasing the ionization efficiency of compounds with high ionization energy and causing ion enhancement [18].

\section{The evaluation of matrix effect}

Matrix effects can dramatically influence analysis performance for both identification and quantification of an analyte. For instance, mass accuracy may deviate due to the matrix effects using high-resolution MS. It can lead to either false negative results due to ion suppression [19] or false positive outputs when, for instance, the signal of the internal standard (IS) undergoes a suppression greater than that of the analyte [4].

The evaluation of matrix effects is explicitly suggested in the US FDA guidelines on bioanalytical analysis [19,20]. There are two main protocols to evaluate the matrix effect: postcolumn infusion protocol and postextraction addition protocol $[10,11,13]$.

\section{Postcolumn infusion protocol}

The postcolumn infusion protocol is carried out using a built-in T-union, syringe pump and LC-pumps as shown in Figure 6A in [21]. The mobile phases are delivered by the LC pumps using a gradient or isocratic program and a standard solution containing the analytes is continuously introduced into the ionization source by a syringe pump connected to the T-union. After injecting a blank sample extract into the LC-MS system, a drop or rise along the 'apparent' baseline indicates suppression or enhancement in ionization of the analyte due to the presence of co-eluting components. If the drop/rise regions are not in the time window of target analytes, it would not influence the analysis of analytes, although the sample matrix causes the ion-suppression or enhancement. Since, total ion chromatogram does not reflect the matrix effect of individual analytes, the extracted signals such as selected ion-monitoring (SIM) or selected reaction monitoring (SRM) should be used to evaluate the matrix effect. When multiple analytes are present, individual analyte should be evaluated to avoid the influence caused by other analytes. A good example is shown in Figure 6B in [21] using the postcolumn infusion protocol to evaluate the matrix effect on Ac-EEMQRR-amide. Serious ion-suppression (red line) was observed in the retention time (RT) window of 5.3-5.5 min, which overlapped the RT of the target analyte, AC-EEMQRR-amide, RT $=5.35 \mathrm{~min}$. Evidently, after hydrophilic interaction LC-solid phase extraction treatment (HILIC-SPE), the matrix effect became negligible because the response (green line) is very close to those in neat solution (blue line).

As shown in Figure 6 of [21], using the postcolumn infusion method can clearly indicate the region in the chromatogram where the analytes and ISs are influenced by matrix effects. Postcolumn infusion can also be used to optimize the mobile phase $\mathrm{pH}$ or additive concentration to promote alternative adduct ion-formation and minimize the matrix effects without dramatically changing the optimized chromatographic conditions [10].

\section{Postextraction addition protocol}

In the case of the postextraction addition method, the matrix effect can be quantitatively evaluated by comparing the response of the analyte in standard solution to that of a postextract spiked with the analyte at the same concentration. The matrix effect can be calculated by the following formula:

$$
\text { Matrix Effect }(M E)=\frac{A-B}{A} \times 100
$$


Where A is the peak area of an analyte in a standard solution (in neat solvents). B is the peak area of the analyte in a sample, which does not contain the analyte and has undergone sample preparation and later spiked with the analyte at the same concentration as the standard solution.

If $\mathrm{ME} \sim 0 \%$, there is no matrix effect. If $\mathrm{ME}>0 \%$, an ion-suppression occurs and, if $\mathrm{ME}<0 \%$, ion-enhancement occurs. In order to get more reliable results, the matrix effect can also be assessed by comparing the slope of a calibration curve for standard solutions with that of matrix matched standard solutions. Lower slope for matrix matched standard solutions suggests ion-suppression while higher slope indicates ion enhancement.

\section{Strategies to minimize/compensate matrix effect}

Although, matrix effects cannot be completely avoided during LC-MS analysis, it can be minimized or compensated for by optimizing sample preparation procedures, and manipulating LC and MS conditions. When the concentration of target analyte is high, reducing the injection volume and/or diluting the sample are the simplest ways to decrease the amount of co-eluting components and minimize the matrix effects. Changing LC conditions, such as, using a lower flow rate, slower gradient program, postcolumn split, a mixed-mode column, micro or nano LC and 2D LC system can also reduce matrix effects [22].

\section{Selection of API ion source \& polarity}

For less polar, volatile and thermally stable analytes, if they can be efficiently ionized through different ionization sources (ESI, APCI and APPI) in either positive or negative mode, APPI and APCI ion sources are preferred. However, APPI source is preferred because it has been shown to be less susceptible to matrix effects compared with APCI and ESI ion sources [18]. Desorption APPI source has even better tolerance over ESI and desorption ESI because the desorption process of Desorption APPI is thermal and only volatile or semivolatile compounds are efficiently evaporated to the gas phase [23]. Sometimes, switching of ionization polarity can also significantly reduce the matrix effects.

\section{The use of internal standard}

Structural analogs and stable isotopically labeled (SIL) compounds are often used as internal standards to compensate matrix effects. Particularly, SIL-ISs are preferred because they have the same structures and similar chromatographic properties as their native compound. SIL-ISs are compounds in which several atoms of the analyte are replaced by their stable isotopes, such as ${ }^{2} \mathrm{H}(\mathrm{D}),{ }^{13} \mathrm{C},{ }^{15} \mathrm{~N}$ or ${ }^{17} \mathrm{O} /{ }^{18} \mathrm{O}$. To avoid that the natural occurrence of analyte isotopes interfere with the labeled IS, the mass difference between the analyte and the corresponding SIL-IS should be at least $3 \mathrm{Da}$ [19]. A SIL-IS may compensate matrix effect, but this approach may not be able to overcome the loss in sensitivity due to the matrix effects. Furthermore, SIL-ISs are costly and not always commercially available and may need to be custom synthesized. Under certain circumstances, deuterated ISs (especially those with higher number of $\mathrm{D}$ atoms) have slight different $\mathrm{RT}$ and do not co-elute with their native analytes and cause inaccuracies in quantification. This phenomenon is induced by deuterium isotope effect due to a small change in lipophilicity [19]. For this reason, more expensive ${ }^{13} \mathrm{C}$-, ${ }^{15} \mathrm{~N}$ - or ${ }^{17} \mathrm{O}$-labeled analogs are considered to be more appropriate than the deuterium labeled isotopes.

\section{Methods of standard addition \& matrix match calibration}

If SIL-ISs are not available, the standard addition could be the most suitable method to compensate matrix effects for complex matrices. However, this approach is laborious and time consuming because a calibration needs to be prepared for each sample. This is almost impracticable for a large number of routine samples with a wide range of concentration. Matrix-matched standards are widely used for bioanalysis in the pharmaceutical industry [20]. However, this method is limited to suitable and representative blank sample/matrix. In addition, standard addition method or matrix-matched calibration curve method will lose sensitivity caused by matrix effects without suitable sample preparation.

\section{Sample preparation \& clean-up}

A comprehensive way to remove co-eluting components is to perform suitable sample preparation and cleanup procedures. Widely used sample preparation methods include partition, filtration, centrifugation, sonication, precipitation, dilution and various forms of extraction. Depending on sample type and analyte properties, liquidliquid extraction (LLE), protein precipitation, solid phase extraction (SPE), and quick, easy, cheap, effective, rugged 
and safe (QuEChERS) [24] are popular sample preparation procedures. With an appropriate absorbent, an SPE procedure may lead to less matrix effects as the interfering matrix can be reduced or eliminated. Automated SPE equipment and online 2D-LC, using a column-switching device, have recently been developed and broadly applied in different fields [25-27]. For 2D-LC, the first column runs SPE which could be reversed phase, HILIC [28-30], porous graphitized carbon LC (PGC-LC) [31], size exclusion or some other modes, including mixed mode columns. The second column performs the regular HPLC separation of the analytes eluted from the first column. With the automation of sample preparation and the advancements in the chromatographic systems, the sample throughputs are significantly increased. When preconcentration of sample is involved, more attention should be paid as this not only increases the concentration of the target analyte but often also enhances the potential interferences. Therefore, the sample preparation may concurrently increase matrix effect [4].

An individual approach to minimize/compensate matrix effect has its own advantages and limitations. For example, some methods need special instrument parts, while others are costly, laborious or time consuming. Based on requirements of specific applications, a few approaches are usually applied in a method. For instance, authors developed an HILIC-MS/MS method using HILIC-SPE and SIL-ISs to determine hexa-peptides in complex matrix for in vitro peptides penetration studies. The method delivered both highly sensitivity and reliable results for the determination of hexa-peptides in human skin, hairless guinea pig skin, cream emulsion and washing fluids containing surfactants [32].

\section{'Comprehensive matrix effect' \& matrix effect factor}

For some studies, such as cosmetic analyses, no representative matrix is available because each product has unique ingredients. Therefore, the calibration standards have to be prepared in neat solvents. In addition, since target analytes in cosmetics are often present in a broad concentration range, a calibration curve to cover a wider range is needed to minimize unnecessary dilution and operational errors. It is obvious that the above protocols for evaluating matrix effect are not suitable and even impractical for the cosmetic analysis. To compensate matrix effects, a corresponding SIL-IS for each analyte (or one SIL-IS for each group of analogs) is extensively used and added in the very beginning of the sample preparation.

Because SIL-ISs are used in each sample, 'comprehensive matrix effect' (CME) is evaluated by comparing the response of individual SIL-IS instead of the analyte in a standard solution to that of a sample at the same concentration. The 'Comprehensive Matrix Effect' is different from matrix effect discussed previously because SILISs are added in the very beginning of the sample preparation procedure. Therefore, CME reflects any loss/gain in both the sample preparation and matrix effect caused by co-eluting components during ionization in an MS ion source. The evaluation of matrix effect and determination for an analyte are combined into one step. The CME concept was initially applied in the determination of peptides in cosmetics by an HILIC-MS/MS method [21]. To differentiate the conventional matrix effect from the 'CME', 'MEF' was defined and introduced in our publication on the determination of cocamidopropyl betaine impurities in cosmetic products [33].

The MEF is calculated as follows:

$$
\operatorname{Matrix} \text { Effect Factor }(\mathrm{MEF})=\frac{[\text { Avg_Area_IS_Stds] }-[\text { Area_IS_Sample }]}{[\text { Avg_Area_IS_Stds }]} \times 100
$$

Where [Avg_Area_IS_Stds] is the averaged peak area of the corresponding SIL-IS in the standards (in neat solutions) and $[$ Area_IS_Sample] $=$ the peak area of the SIL-IS in a sample.

If $\mathrm{MEF} \sim 0 \%$, there is no CME, that is, there is negligible loss/gain in both the sample preparation and ionization. If an absolute MEF of a sample is $\leq$ the threshold, which is decided during the method validation, the result of the sample is acceptable with no further action. To simplify the calculation, an excel template was developed by the authors to calculate MEFs and a result table was generated from the processed data. Based on our experiences, the result of a sample was considered acceptable if its MEF was $\leq 85 \%$ [33]. Otherwise, the sample was noted as 'not quantifiable' which indicates that this sample needed to be diluted or further sample preparation was needed to minimize the matrix effect. The results table includes the analyte amount for each sample, RSDs (for replicated 
samples only), MEFs and confirmation ion ratio, which is calculated below:

$$
\text { Ion ratio }=\frac{\text { Peak Area (confirmatio ion) }}{\text { Peak Area (quantitation ion) }} \times 100
$$

Where peak area (confirmation ion) is the confirmation SRM transition (secondary SRM transition) peak area of an analyte in a standard or a sample, and peak area (quantitation ion) = the quantitation SRM transition (primary SRM transition) peak area of the analyte in the standard or the sample. The MEF was successfully applied to our previous study for the determination of cocamidopropyl betaine impurities in cosmetic products [33].

\section{Conclusion}

Matrix effects are major concerns in quantitative LC-MS analysis because they detrimentally affect the accuracy, precision and sensitivity of a method. Therefore, the presence of matrix effects needs to be thoroughly evaluated during the method development to achieve reliable analytical data. The evaluation of matrix effects and strategies to minimize/compensate matrix effects have been comprehensively discussed. Here, we have also discussed in details the concept of MEF, demonstrated its use to determine matrix effects and its importance in development of reliable and accurate LC-MS methods for quantitative analysis.

\section{Acknowledgements}

The authors wish to thank R Pawar for reviewing the manuscript.

\section{Financial \& competing interests disclosure}

The authors have no relevant affiliations or financial involvement with any organization or entity with a financial interest in or financial conflict with the subject matter or materials discussed in the manuscript. This includes employment, consultancies, honoraria, stock ownership or options, expert testimony, grants or patents received or pending, or royalties.

No writing assistance was utilized in the production of this manuscript.

\section{References}

1. Matuszewski B, Constanzer M, Chavez-Eng C. Matrix effect in quantitative LC/MS/MS analyses of biological fluids: a method for determination of finasteride in human plasma at picogram per milliliter concentrations. Anal. Chem. 70(5), 882-889 (1998).

2. Matuszewski B, Constanzer M, Chavez-Eng C. Strategies for the assessment of matrix effect in quantitative bioanalytical methods based on HPLC-MS/MS. Anal. Chem. 75(13), 3019-3030 (2003).

3. Kebarle P, Tang L. From ions in solution to ions in the gas phase - the mechanism of electrospray mass spectrometry. Anal. Chem. 65(22), A972-A986 (1993).

4. Gosetti F, Mazzucco E, Zampieri D, Gennaro MC. Signal suppression/enhancement in high-performance liquid chromatography tandem mass spectrometry. J. Chromatogr. A 1217(25), 3929-3937 (2010).

5. Ikonomou MG, Blades AT, Kebarle P. Investigations of the electrospray interface for liquid chromatography/mass spectrometry. Anal. Chem. 62(9), 957-967 (1990).

6. Ghosh C, Shinde CP, Chakraborty BS. Influence of ionization source design on matrix effects during LC-ESI-MS/MS analysis. J. Chromatogr. B 893, 193-200 (2012).

7. Cech NB, Enke CG. Practical implications of some recent studies in electrospray ionization fundamentals. Mass Spectrom. Rev. 20(6), 362-387 (2001).

8. Trufelli H, Palma P, Famiglini G, Cappiello A. An overview of matrix effects in liquid chromatography-mass spectrometry. Mass Spectrom. Rev. 30(3), 491-509 (2011).

9. Panuwet P, Hunter RE Jr, D’Souza PE et al. Biological matrix effects in quantitative tandem mass spectrometry-based analytical methods: advancing biomonitoring. Crit. Rev. Anal. Chem. 46(2), 93-105 (2016).

10. Furey A, Moriarty M, Bane V, Kinsella B, Lehane M. Ion suppression; a critical review on causes, evaluation, prevention and applications. Talanta 115, 104-122 (2013).

11. Yaroshenko D, Kartsova L. Matrix effect and methods for its elimination in bioanalytical methods using chromatography-mass spectrometry. J. Anal. Chem. 69(4), 351-358 (2014).

12. Jessome LL, Volmer DA. Ion suppression: a major concern in mass spectrometry. LCGC North America 24(5), 498-510 (2006).

13. King R, Bonfiglio R, Fernandez-Metzler C, Miller-Stein C, Olah T. Mechanistic investigation of ionization suppression in electrospray ionization. J. Am. Soc. Mass Spectrom. 11(11), 942-950 (2000). 
14. Hanold KA, Fischer SM, Cormia PH, Miller CE, Syage JA. Atmospheric pressure photoionization. 1. General properties for LC/MS. Anal. Chem. 76(10), 2842-2851 (2004).

15. Takino M, Daishima S, Nakahara T. Liquid chromatography/mass spectrometric determination of patulin in apple juice using atmospheric pressure photoionization. Rapid Commun. Mass Spectrom. 17(17), 1965-1972 (2003).

16. Theron HB, van der Merwe MJ, Swart KJ, van der Westhuizen JH. Employing atmospheric pressure photoionization in liquid chromatography/tandem mass spectrometry to minimize ion suppression and matrix effects for the quantification of venlafaxine and O-desmethylvenlafaxine. Rapid Commun. Mass Spectrom. 21(10), 1680-1686 (2007).

17. Prakash C, Shaffer CL, Nedderman A. Analytical strategies for identifying drug metabolites. Mass Spectrom. Rev. 26(3), 340-369 (2007).

18. da Silveira GD, Faccin H, Claussen L et al. A liquid chromatography-atmospheric pressure photoionization tandem mass spectrometric method for the determination of organosulfur compounds in petroleum asphalt cements. J. Chromatogr. A1457, 29-40 (2016).

19. Van Eeckhaut A, Lanckmans K, Sarre S, Smolders I, Michotte Y. Validation of bioanalytical LC-MS/MS assays: evaluation of matrix effects. J. Chromatogr. B 877(23), 2198-2207 (2009).

20. EMA. Bioanalytical Method Validation: Guidance for Industry. www.fda.gov/downloads/Drugs/Guidances/ucm070107.pdf

21. Zhou W, Wang PG, Krynitsky AJ, Rader JI. Rapid and simultaneous determination of hexapeptides (Ac-EEMQRR-amide and H 2 N-EEMQRR-amide) in anti-wrinkle cosmetics by hydrophilic interaction liquid chromatography-solid phase extraction preparation and hydrophilic interaction liquid chromatography with tandem mass spectrometry. J. Chromatogr. A 1218(44), 7956-7963 (2011).

22. Lien G-W, Chen C-Y, Wang G-S. Comparison of electrospray ionization, atmospheric pressure chemical ionization and atmospheric pressure photoionization for determining estrogenic chemicals in water by liquid chromatography tandem mass spectrometry with chemical derivatizations. J. Chromatogr. A 1216(6), 956-966 (2009)

23. Suni NM, Lindfors $\mathrm{P}$, Laine $\mathrm{O}$ et al. Matrix effect in the analysis of drugs of abuse from urine with desorption atmospheric pressure photoionization-mass spectrometry (DAPPI-MS) and desorption electrospray ionization-mass spectrometry (DESI-MS). Anal. Chim. Acta 699(1), 73-80 (2011).

24. Anastassiades M, Lehotay SJ, Stajnbaher D. Fast and easy multiresidue method employing acetonitrile extraction/partitioning and "dispersive solid-phase extraction" for the determination of pesticide residues in produce. J. AOAC Int. 86(2), 412-431 (2003).

25. Holm A, Storbråten E, Mihailova A, Karaszewski B, Lundanes E, Greibrokk T. Combined solid-phase extraction and 2D LC-MS for characterization of the neuropeptides in rat-brain tissue. Anal. Bioanal. Chem. 382(3), 751-759 (2005).

26. Oliveira RV, Henion J, Wickremsinhe ER. Automated direct extraction and analysis of dried blood spots employing on-line SPE high-resolution accurate mass bioanalysis. Bioanalysis 6(15), 2027-2041 (2014).

27. Yang S, Toghi Eshghi S, Chiu H, DeVoe DL, Zhang H. Glycomic analysis by glycoprotein immobilization for glycan extraction and liquid chromatography on microfluidic chip. Anal. Chem. 85(21), 10117-10125 (2013).

28. Wilson SR, Jankowski M, Pepaj M et al. 2D LC separation and determination of bradykinin in rat muscle tissue dialysate with on-line SPE-HILIC-SPE-RP-MS. Chromatographia 66(7-8), 469-474 (2007).

29. Buszewski B, Noga S. Hydrophilic interaction liquid chromatography (HILIC) - a powerful separation technique. Anal. Bioanal. Chem. 402(1), 231-247 (2012).

30. Wang PG, He W. Aqueous normal-phase chromatography: the bridge between reversed-phased and HILIC. In: Hydrophilic Interaction Liquid Chromatography (HILIC) And Advanced Applications. Wang PG, He W (Eds). CRC Press, NY, USA (2011).

31. Rogeberg M, Malerod H, Roberg-Larsen H, Aass C, Wilson SR. On-line solid phase extraction-liquid chromatography, with emphasis on modern bioanalysis and miniaturized systems. J. Pharm. Biomed. Anal. 87, 120-129 (2014).

32. Zhou W, Wang PG, Ogunsola OA et al. Rapid determination of hexapeptides by hydrophilic interaction LC-MS/MS for in vitro skin-penetration studies. Bioanalysis 5(11), 1353-1362 (2013)

33. Wang PG, Zhou W. Rapid determination of cocamidopropyl betaine impurities in cosmetic products by core-shell hydrophilic interaction liquid chromatography-tandem mass spectrometry. J. Chromatogr. A 1461, 78-83 (2016). 